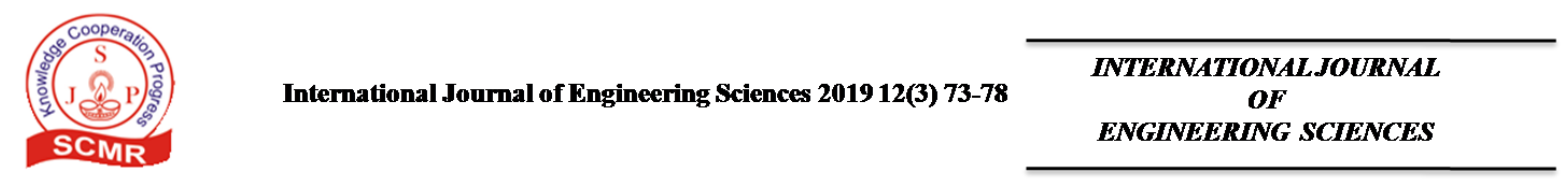

\title{
Effect of unit cell shape and strut size on flexural properties of ordered Copper foam
}

\author{
Pulak M Pandey ${ }^{*}$ and Gurminder Singh \\ Mechanical Engineering Department, Indian Institute of Technology Delhi, New Delhi, India
}

(Received 31 July 2019, accepted 30 September 2019)

https://doi.org10.36224/ijes.120301

\begin{abstract}
The effect of unit cell shape and strut size variation was studied on the flexural properties of the ordered copper foam in the present study. The developed rapid manufacturing method using 3D printing and ultrasonic vibrationassisted pressureless sintering was employed to fabricate samples. Two different shapes such as simple cubic (SC) and body-centred cubic (BCC) were used as the unit cells for the samples with three different strut sizes 1, 1.3 and $1.6 \mathrm{~mm}$. Three-point bend test was performed to obtain the flexural properties such as flexural modulus and ultimate flexural strength. Different shapes of failure curves were noticed during the test, which was owing to the different unit cell shapes. The fracture was obtained to be brittle in nature due to the micro-pores of the struts. The fractured surfaces of the two different unit cells were also studied. The samples with the simple cubic unit cell shapes possessed better flexural properties as compared to the body-centred cubic unit cell samples.
\end{abstract}

Keywords: 3D printing, copper, ordered foam, flexural properties, sintering

\section{Introduction}

High specific area along with specific strength are the unique properties of metal foams, which have increased the metal foam interest at industrial level in recent years. These metal foams have a high impact in different application sectors such as aerospace, automobile, heat exchangers, biomedical, etc [1]. Generally, these foams are classified into two categories on the basis of pores nature- open and closed metal foams. Closed porous metal foams are highly used in the impact-absorbing applications. However, with an open-cell structure, metal foams have a huge impact on the heat exchanger applications [2]. Foam open-cell nature improves the heat transfer in the endothermic and exothermic reactions and also have sufficient mechanical strength such as compression and bending strength to use in catalytic reactors, gas purifications system, and future mechanical thermal systems. Uniform open-cell foams have high surface area and play a crucial role in low-pressure drop properties due to uniform pore size distribution and also possess better mechanical properties. High thermal and electrical conductivity with good mechanical properties has enhanced the ability of pure copper to use in mechanical thermal management systems. However, the fabrication methodology of the ordered open-cell copper foams was found to be limited in the literature.

Generally, metal additive manufacturing techniques like selective laser melting (SLM), electron beam melting (EBM), direct metal laser sintering (DMLS), etc. were used to fabricate metal foams. However, the fabrication of copper foams was difficult using these traditional additive manufacturing techniques. Laser during scanning of the metal powder reflected rapidly owing to the large coefficient 
ofthermal conductivity of copper in the DMLS process [3]. It resulted in the poor fabrication of copper metal foams with curling, swelling, and other defects. Use of electron beam in place of laser in EBM technique minimized the optical reflectivity. Yang et al. [4] and Ramirez et al. [5] has reported successful attempts to fabricate uniform copper open-cell foam using EBM. However, the fabricated foams from the EBM process possessed more than $12 \%$ shrinkage. Recently, a novel rapid tooling approach with the amalgamation of 3D printing and ultrasonic vibration-assisted sintering was reported for the fabrication of copper open-cell foams [6,7]. The optimization parameters to acquire the maximum density and minimum shrinkage was obtained to fabricate metal foams [8]. It was observed that the process was able to fabricate uniform copper metal foams with different unit cell shape having maximum of $8.07 \%$ shrinkage.

Metal foam strut size and unit cell shape are the important parameters to acquire better strength with low weight. Sharma and Pandey [9] reported enhancement in the mechanical properties like compression and bending properties of open-cell iron foam with increment in strut size. Similarly, Rashid et al. [10] reported the importance of unit cell shape on the bending properties of aluminium metal foam. Tian et al. [11] also reported the unit cell shape effect on the bending behaviour of the open-cell foam during the flexural test with different porosities.

From the literature, it is evident that the copper open-cell uniform foams have application in the thermal management system due to its parent material high thermal conductivity and mechanical strength. Few attempts $[4,5]$ were reported to fabricate uniform copper metal foams. However, the use of UAPS and 3D printing has shown promising efforts to fabricate copper foams with less shrinkage [8]. Metal foams with different unit cell shape and strut size haveshown different bendingbehaviour during the flexural test. Therefore, an attempt has been made to evaluate the unit cell shape and strut variations effect on the flexural properties of the ordered copper foam. The developed rapid tooling method using 3D polymer printer and ultrasonic assisted sintering was employed to fabricate simple cubic (SC) and body centered cubic (BCC) unit cell shape felexural samples with different sizes. Flexural properties were evaluted along with fracture behaviour and energy absorption capacity.

\section{Material and methods}

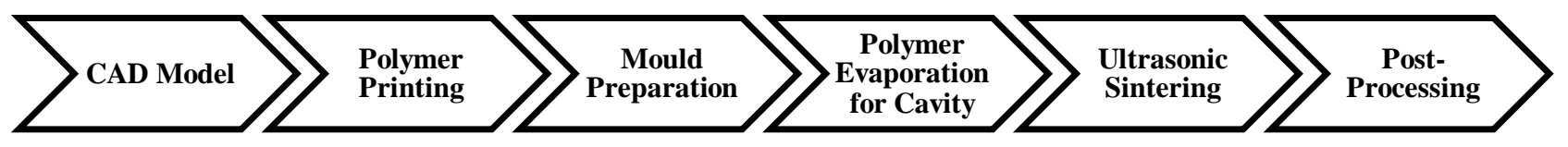

Figure 1: Flow chart of the adopted rapid manufacturing process

Spherical shape copper powderwith average particle size of $8 \mu \mathrm{m}$ was used for rapid manufacturing. The amalgamation of ultrasonic vibration-assisted sintering and polymer 3D printing was used as the rapid toolingtechnique for specimen fabrication. The flow diagram of the rapid manufacturing process is shown in Fig. 1. First, the specimens for the flexural test of size $100 \mathrm{~mm} \times 12 \mathrm{~mm} \times 6 \mathrm{~mm}$ were modelled in the CREO software. Two different shapes, Simple cubic (SC) and body-centred cubic (BCC) unit cell shapes with three different strut sizes of $1.0,1.3$ and $1.6 \mathrm{~mm}$ were used to study the unit cell shape and strut size effects. Stereolithography based polymer 3D printerwas employed to manufacture polymer parts after tessellation and slicing. Polymer printed parts were used as thepattern in rapid tooling technique to prepare a mould cavity. A steel crucible was used to prepare the mould. After the settlement of the investment powder, the crucible was placed in a furnace for polymer pattern evaporation and baking of investment powder. Further, mould cavity crucible was assembledwith the customized design ultrasonic horn and furnace [12]. The metal powder was poured in the cavity and sintered 
at $809^{\circ} \mathrm{C}$ sintering temperature with $4.6^{\circ} \mathrm{C} / \mathrm{min}$ heating rate, 52 min soaking time and $86 \%$ ultrasonic power intensity. The sintering cycle was obtained by optimizing the parameters to maximize the density, minimizeshrinkage and maximize the compressive strength for the pure copper solid cylindrical specimens in a previous study [8]. After sintering, the specimens were cleaned using ultrasonicationin the acetone along with shot blasting treatment. The detail description of the adopted rapid manufacturing technique is given by Singh and Pandey [7].

Instron universal testing machine with $100 \mathrm{kN}$ capacity was employed for the flexural test of the fabricated samples. The test was performed with the $1 \mathrm{~mm} / \mathrm{min}$ constant traverse velocity. The flexural properties such as ultimate flexural strength and modulus of elasticity were calculated from the stress-strain graphs. The following equations were used to calculate the flexural properties:

$$
\sigma_{f}=\frac{3 \times F \times l}{2 \times b \times d^{2}}, \varepsilon_{f}=\frac{6 \times D \times d}{l^{2}}, E_{f}=\frac{l^{3} \times m}{4 \times b \times d^{3}}
$$

where, $\boldsymbol{\sigma}_{\boldsymbol{f}}$ and $\boldsymbol{\varepsilon}_{\boldsymbol{f}}$ are flexural stress and strain, Fand $l$ are applied load and span length, $b$ and $d$ are samples width and thickness. The slope $m$ was determinedto the tangent of the forcedisplacement curve.

\section{Results and discussion}

Figure 2(a) depicted the fabricated flexural test samples. The adopted rapid manufacturing method was efficient tomanufacture copper foams long length flexural sample with no pore blockage. SEM image of the strut of the fabricated foam is shown in Fig. 3. Isolated micropores were observed, which signified the efficient diffusion of the copper powder particles [13]. Further, the fabricated samples were used on the UTM machine for the flexural test as shown in Fig. 2(b).

(a)
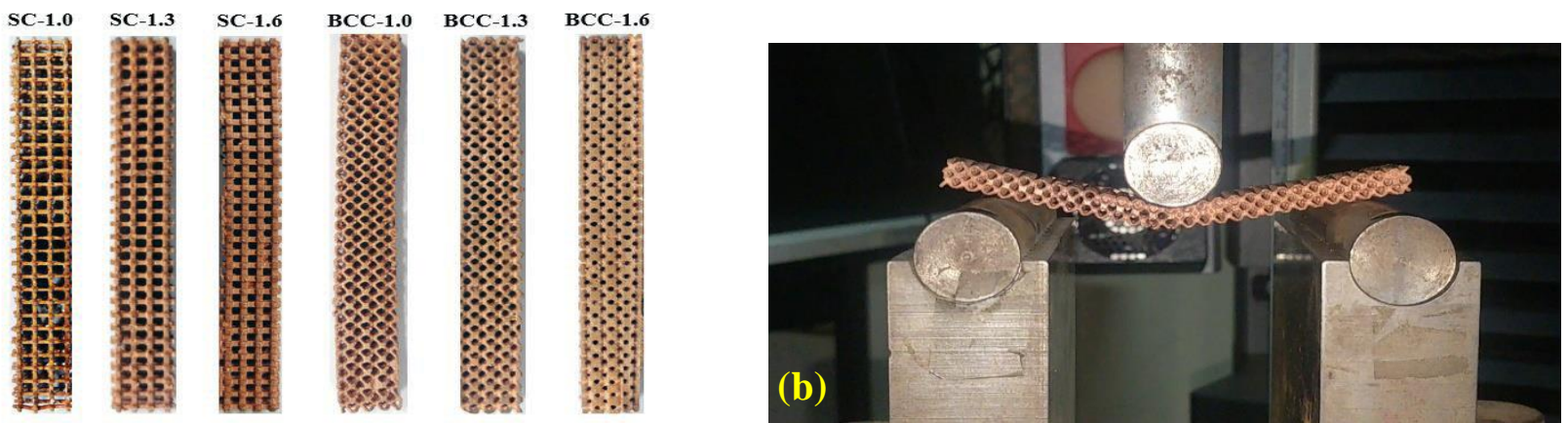

Figure 2: (a) Fabricated flexural specimens by adopted rapid manufacturing of SC and BCC foams and (b) flexural experiment on Instron UTM

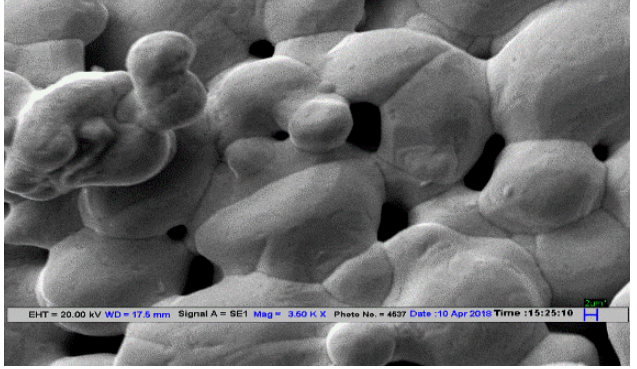

Figure 3: SEM image of strut of the fabricated foam 

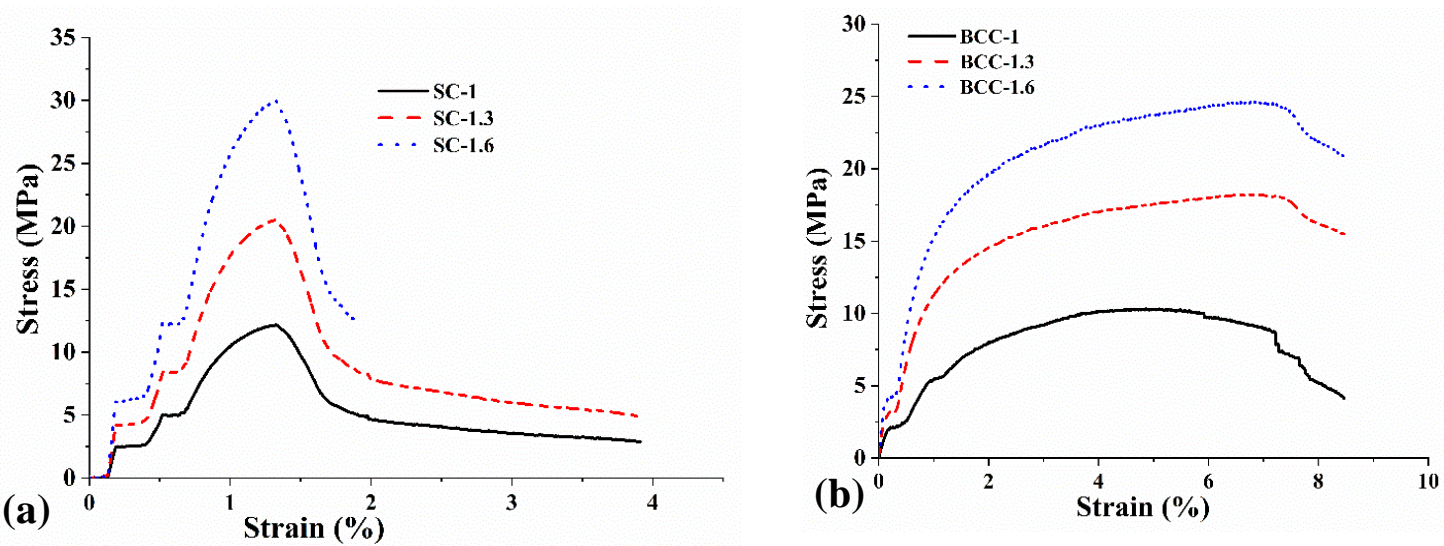

Figure 4: Flexural stress-strain graphs for (a) SC and (b) BCC flexural samples with different strut sizes

Figure 4 depicted the flexural stress-strain graph for SC and BCCflexural samples. It was noticed from the graphs that both unit cell shapes fabricated foams possessed plastic deformation before failure. High amount of hardening was noticed in the BCC foams before failure. However, copper is ductile in nature but owing to the presence of micro-pores,fracture started from the pores and led to brittle behaviour.A bell shape stress-strain curve was observed in the SC foam with a shorter plateau region with three different sturt sizes. In BCC foam, the stress increased constantly and possessed wider plateau range with different strut sizes. It was due to the different shapes of the unit cells. In SC, the struts were perpendicular to each other. Consequently, in BCC, the struts were inclined at $45^{\circ}$ and the load was shared by the two struts and sustained stress for a longer time. Figure 5 represents the SEM observations of the strut of the SC and BCC flexural samples after the fracture. The images were examined in order to identifythe flexural nature of the fabricated samples. The fracture found to start at the powder particles diffusion joints. The diffusion neck between the powder particles wasfailed after the applied load surpasses the bending fracture limit. Effect of different unit cell shapes was noticed in the fractured images. Rough dimples were evaluated in SC foam and smooth dimples were noticed in the BCC foam.
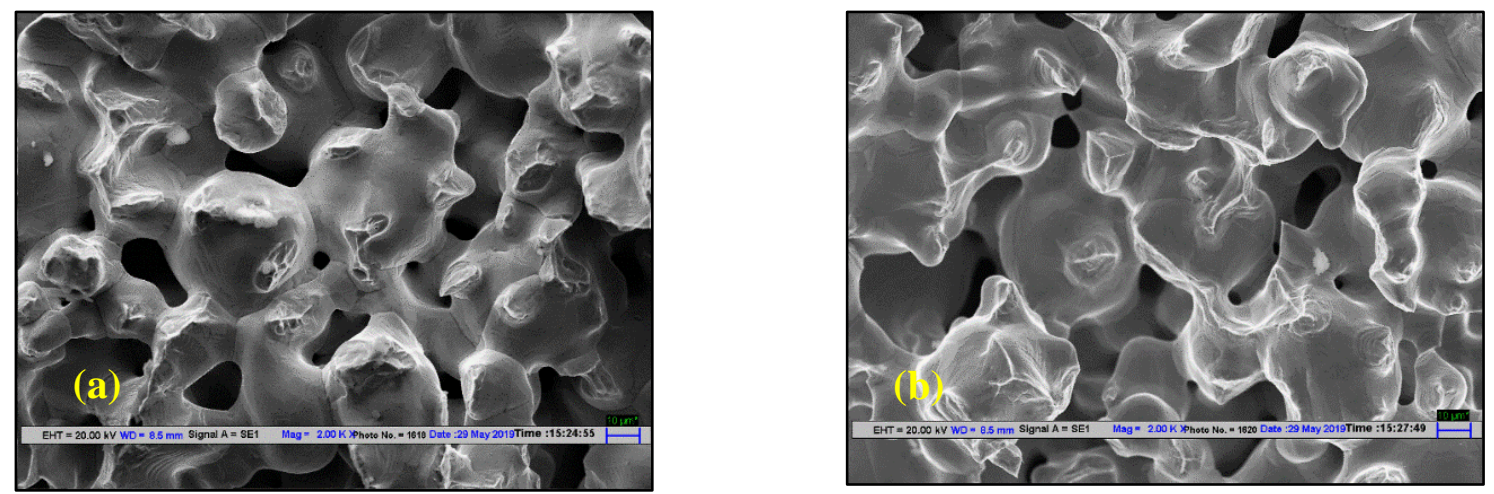

Figure 5: SEM observations of (a) SC and (b) BCC flexural samples after bending test

Figure 6 depicted the strut size variation effect for the flexural properties such as flexural modulus along with ultimate flexural strength for SC and BCC foams. It was noticed that both properties flexural modulus of elasticity and ultimate flexural strength improved with increment in strut size. It was due to the increment in density with increasing strut size [9]. Also, the SC unit cell shape samples possessed high 
flexural strength and flexural modulus as compared BCC unit cell shape. It was due to the high relative density and unit cell shape of SC samples [6]. The flexural modulus along with flexural strength increased with high margin in SC samples as compared BCC foam samples which was due to the unit cell shape difference as discussed above.
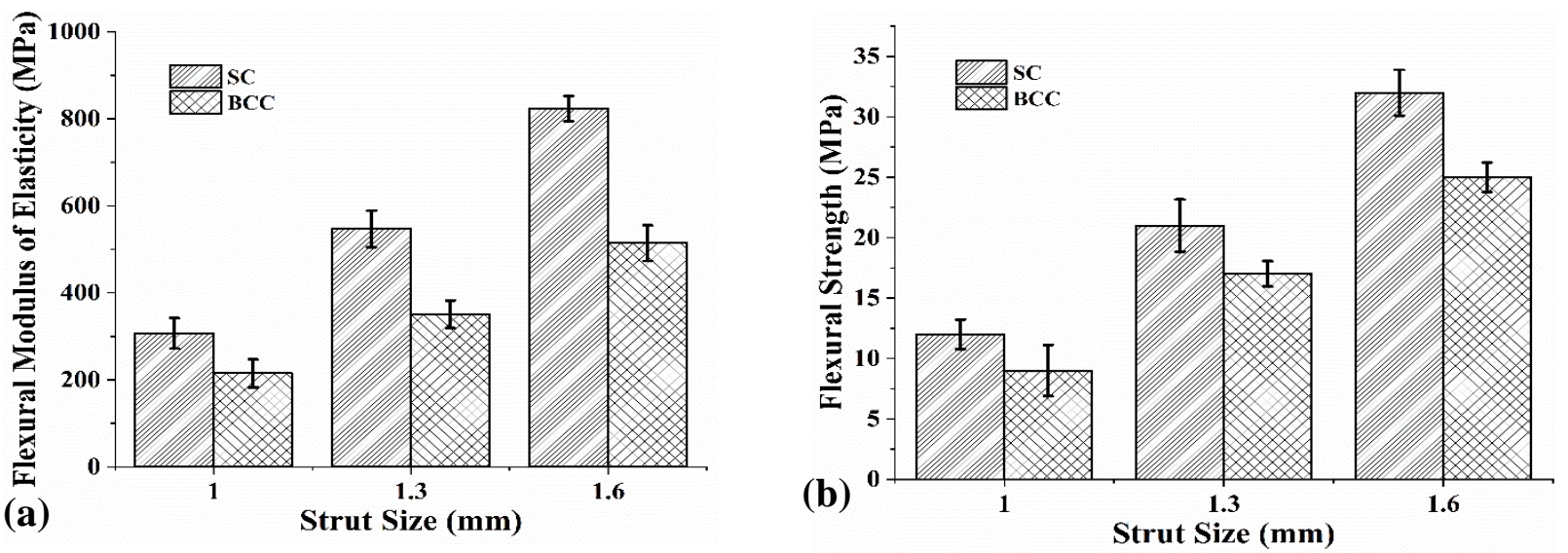

Figure 6: (a) Flexural modulus along with (b) ultimate flexural strength for SC and BCC unit cell foam with different strut sizes

The flexural energy absorption capacity of the samples was also evaluated by calculating the area under the stress-strain curve upto the fracture limit. It was observed that SC foam samples exhibited $1-12 \mathrm{NJ} / \mathrm{m}^{3}$ energy absorption capacity and was higher as compared to BCC foams samples with $2-8 \mathrm{NJ} / \mathrm{m}^{3}$ energy absorption capacity for three strut sizes. However, the BCC foams possessed high flexural plateau stress as compared to SC foams samples.

\section{Conclusion}

The effect of different unit cell shapes and different strut sizes were evaluated on the flexural properties of the ordered copper foam fabricated by the amalgamation of 3D printing and ultrasonicibration-assistedsintering. Following inference were drawn from the present work.

- The established process was efficient to manufacture long flexural ordered copper foam specimens with no blockage of pores and different unit cells with strut size variations.

- Brittle behaviour of the foam was noticed in contradictory to the copper material ductile nature. It was owing to the micropores formation in the foam struts due to pressureless sintering and crack generated from the pores and resulted in brittle fracture.

- Simple cubic (SC) unit cell shape samples exhibited bell shape stress-strain curve with a shorter plateau area as compared to body-centred cubic (BCC) unit cell samples. However,SC samples possessed high energy absorption capacity of $1-12 \mathrm{NJ} / \mathrm{m}^{3}$ as compared to BCC samples of $2-8 \mathrm{NJ} / \mathrm{m}^{3}$.

- Rough dimples were observed in SC samples as compared to BCC samples during the necks study after the fracture.

- The thick struts size samples possessed high strength properties for both types of unit cell shapes. The SC unit shape flexural samples showed the high flexural modulus (310-843 $\mathrm{MPa}$ ) and flexural strength (14-32 $\mathrm{MPa}$ ) as compared to the $\mathrm{BCC}$ samples flexural modulus (230-515 MPa) and flexural strength (8-22 MPa). 


\section{References}

1. Ashby M.F., Evans T., Fleck N. A., Hutchinson J. W., Wadley H. N., Gibson L. J., "Metal Foams : A Design Guide", 2000

2. Skibinski J., Cwieka K., Kowalkowski T., Wysocki B., Wejrzanowski T., Kurzydlowski K. J., "The influence of pore size variation on the pressure drop in open-cell foams". Materials and Design, 2015, 87, 650-655

3. Pogson S. R., Fox P., Sutcliffe C. J., O'Neill W., "The production of copper parts using DMLR", Rapid Prototyping Journal, 2003, 9, 5, 334-343

4. Yang L., Harrvsson O., West H., Cormier D., "Design and characterization of orthotropic re-entrant auxetic structures made via EBM using Ti6Al4V and pure copper", International Solid Freeform Fabrication Symposium, 2011 , 464-474

5. Ramirez D. A., Murr L. E., Li S. J., Tian Y. X., Martinez E., Martinez J. L., Machado B. I., Gaytan S. M., Medina F., Wicker R. B., "Open-cellular copper structures fabricated by additive manufacturing using electron beam melting", Materials Science and Engineering A, 2011, 528, 16-17, 5379-5386.

6. Singh G., Pandey P. M., "Uniform and graded copper open cell ordered foams fabricated by rapid manufacturing: surface morphology , mechanical properties and energy absorption capacity", Materials Science \& Engineering A, 2019, 761, 138035

7. Singh G., Pandey P. M., "Ultrasonic Assisted Pressureless Sintering for rapid manufacturing of complex copper components", Materials Letters, 2019, 236, 276-280

8. Singh G., Pandey P. M., "Rapid manufacturing of copper components using $3 D$ printing and ultrasonic assisted pressureless sintering: experimental investigations and process optimization", Journal of Manufacturing Processes, 2019, 43, 253-269

9. Sharma P., Pandey P. M., "Morphological and mechanical characterization of topologically ordered open cell porous iron foam fabricated using 3D printing and pressureless microwave sintering", Materials \& Design, 2018, 160, 442-454

10. Rashid RA. R., Mallavarapu J., Palanisamy S., Masood S. H., "Comparative study of flexural properties of additively manufactured aluminium lattice structures", Materials Today: Proceedings, 2017, 4, 8, 8597-8604

11. Tian .C, Li .X, Li .H, Guo .G, Wang .L, Rong .Y, "The effect of porosity on the mechanical property of metal-bonded diamond grinding wheel fabricated by selective laser melting ( SLM )", Materials Science \& Engineering A, 2019, 743, 697-706

12. Singh G., Pandey P. M., "Design and Analysis of Long-Stepped Horn for Ultrasonic Assisted Sintering", 21st International Conference on Advances in Materials and Processing Technology (AMPT), 2018

13. German R., "Sintering: From Empirical Observations to Scientific Principles", Sintering: From Empirical Observations to Scientific Principles, 2014 